\title{
The role of nutraceuticals in the prevention of cardiovascular disease
}

\author{
Bozena Sosnowska ${ }^{1}$, Peter Penson ${ }^{2}$, Maciej Banach ${ }^{1,3}$ \\ ${ }^{1}$ Department of Hypertension, Chair of Nephrology and Hypertension, Medical University of Lodz, Lodz, Poland; ${ }^{2}$ School of Pharmacy and \\ Biomolecular Sciences, Liverpool John Moores University, Liverpool, UK; ${ }^{3}$ Polish Mother's Memorial Hospital Research Institute (PMMHRI), \\ Lodz, Poland \\ Contributions: (I) Conception and design: M Banach; (II) Administrative support: P Penson, M Banach; (III) Provision of study materials or patients: \\ M Banach; (IV) Collection and assembly of data: B Sosnowska, M Banach; (V) Data analysis and interpretation: B Sosnowska, P Penson, M Banach; (VI) \\ Manuscript writing: All authors; (VII) Final approval of manuscript: All authors. \\ Correspondence to: Prof. Maciej Banach, MD, PhD, FNLA, FAHA, FESC. Department of Hypertension, WAM University Hospital in Lodz, Medical \\ University of Lodz, Zeromskiego 113, 90-549 Lodz, Poland. Email: maciejbanach@aol.co.uk.
}

\begin{abstract}
Cardiovascular disease (CVD) ranks among the most common health-related and economic issues worldwide. Dietary factors are important contributors to cardiovascular risk, either directly, or through their effects on other cardiovascular risk factors including hypertension, dyslipidemia and diabetes mellitus. Nutraceuticals are natural nutritional compounds, which have been shown to be efficacious in preventative medicine or in the treatment of disease. Several foods and dietary supplements have been shown to protect against the development of CVD. The aim of this review is to present an update on the most recent evidence relating to the use of nutraceuticals in the context of the prevention and treatment of CVD.
\end{abstract}

Keywords: Nutraceuticals; cardiovascular disease (CVD); polyphenols

Submitted Jan 29, 2017. Accepted for publication Feb 27, 2017.

doi: $10.21037 / \mathrm{cdt} .2017 .03 .20$

View this article at: http://dx.doi.org/10.21037/cdt.2017.03.20

\section{Introduction}

Cardiovascular disease (CVD) is common, indeed the majority of adults above sixty years of age will experience some manifestation of CVD. Based on data from 2012 and 2013, it has been estimated that CVD is responsible for 17.3 million deaths annually worldwide (1). Morbidity is also high, and in Europe, 200 billion Euros of healthcare expenditure is attributable to CVD (2). Risk factors for CVD can be categorized as modifiable and non-modifiable. Modifiable risk factors include obesity, hypertension, hyperlipidemia, diabetes mellitus, metabolic syndrome and lifestyle risk factors such as unhealthy diet, smoking and physical inactivity. Dietary factors are also important contributors to cardiovascular risk, either directly, or through their effects on other risk factors including hypertension, dyslipidemia and diabetes mellitus (3). Reduction of risk factors in the population, especially blood pressure reduction and lipid-lowering can have important impacts upon mortality from CVD (4).

Protective effects against CVD have been demonstrated for several foods and dietary supplements (5) thus presenting new possibilities for population-level reduction of CVD risk. Evidence suggests that this approach is very promising. For example, in the PREDIMED observational study, participants in the highest quintile of polyphenol consumption had a relative risk of CVD of $54 \%$ compared to those in the lowest quintile (6). The aim of this review is to present an update on the most recent evidence relating to the use of nutraceuticals in the context of the prevention and treatment of CVD. Unfortunately, few studies have measured the associations between nutraceutical consumptions and "hard" outcomes such as mortality. Large randomized controlled trials are particularly rare, and thus there is a paucity of evidence in this area. Thus, our discussion will be largely focused on the effects of 
nutraceuticals on well-characterized risk factors for CVD.

\section{Nutraceuticals}

The term "nutraceuticals" was introduced by Stephen DeFelice, founder and chairman of the Foundation for Innovation in Medicine, in 1989. A nutraceutical is defined as a "food, or parts of a food, that provide medical or health benefits, including the prevention and treatment of disease" (7). The definition encompasses medicinal products made from natural ingredients. Several classes of nutraceuticals have been proposed to have potential benefits in the treatment of CVD and the ones with the strongest evidence are briefly summarized below.

\section{Sterols/stanols}

Plant sterols/stanols are phytosterols, and have been identified in a range of plant products including various fruits and vegetables, cereals, seeds and nuts. Their biological activity results from their molecular structural similarity to cholesterol (8).

\section{Polyphenols}

Polyphenols are phytochemicals with widespread distribution in foods of plant origin. They are found in fruits, vegetables, cereal and legumes. Additionally, they are found in beverages produced from plant products such as tea, coffee, wine and cocoa. Polyphenols are structurally diverse, and over 8,000 have been identified. These include flavonoids, phenolic acids, stilbenes and lignans (9). Polyphenols found in grapes and grape derivatives, cocoa and tea are of interest in the prevention of CVD. Phenolic compounds are found in grapes and these include anthocyanins, flavanols, flavonols, stilbenes and phenolic acids (10). Resveratrol (3,5,4'-trihydroxy-transstilbene) is the most extensively studied grape-derived stilbene contained mainly in grapes. However, resveratrol is common to a variety of species including cranberries, blueberries, peanuts, and Japanese knotweed (11).

Derivatives of cocoa beans (Theobroma cacao) are widely consumed in cocoa and chocolate (12). A variety of polyphenols have been identified in cocoa and its derivative. These include catechins, flavonol glycosides, anthocyanins and procyanidins (13). Cocoa-containing foods provide a higher content of flavonoids per serving than other beverages such as red wine and tea (14).
The very widespread and frequent consumption of tea makes investigation of its nutraceutical propertied essentials. Polyphenols found in tea include catechins, theaflavins, tannins, and flavonoids. The degree of fermentation of tea leaves influences the chemical composition. Green tea, which is minimally fermented, contains more catechins such as epigallocatechin gallate, epicatechin-3-gallate, epigallocatechin and epicatechin (15) whereas the more extensively fermented black tea is rich in flavins and thearubigins (16).

\section{Spirulina}

Spirulina is a blue-green microalga (Cyanobacterium). Spirulina is a rich source of protein, vitamins, minerals, carotenoids, and phycocyanins and has a very long history of use as a human foodstuff with no apparent concerns over safety $(17,18)$.

Table $S 1$ summarizes the methodology and main findings from each study described in the paper.

\section{Dyslipidemia}

Dyslipidemia is an umbrella term for a variety of lipid abnormalities, which increase the risk of CVD. Reduction of total cholesterol (TC) and low-density lipoproteincholesterol (LDL-C) is effective in the primary and secondary prevention of CVD events (76). In particular, low LDL-C levels are associated with lower rates of major coronary events (77). Thus nutraceuticals with the potential to modify the plasma lipid profile have the potential to reduce the burden of CVD (78). Evidence related to the lipid-modifying effects of nutraceuticals is summarized below.

\section{Sterols/stanols}

Consumption of plant sterols/stanols has been shown to be associated with lower circulating concentrations of TC in humans $(79,80)$. Their effect is predominantly LDL-C reduction with little or no effect on high-density lipoprotein cholesterol (HDL-C) or triglycerides (81). The mechanism by which sterols/stanols reduce LDL-C is associated with a reduction in the intestinal absorption of cholesterol, the upregulation of hepatic LDL receptors (and consequent increased hepatic cholesterol uptake) and reduced production of endogenous cholesterol (82).

Circulating LDL-C concentrations are inversely 
correlated with the extent of sterol/stanol consumption. The reduction in plasma LDL-C concentrations associated with sterol/stanol consumption may be as large as $10 \%$ $(19,20)$. This could lead to reductions in CVD if the effect is associated with a reduction in cardiovascular events similar to that induced by other drugs with similar lipidlowering efficacy. As sterols/stanols reduce the intestinal absorption of cholesterol, their effect may be additive to that of statins which act by the reduction of hepatic cholesterol production. Importantly, a meta-analysis of 15 randomized controlled trials would appear to suggest that this is the case. It was found that a combination of statins and stanols/sterols lowered the levels of TC and LDL-C to a greater extent than with statins alone. HDL-C and triglyceride concentrations were not altered by the addition of sterols/stanols to statin therapy (21). Another metaanalysis demonstrated that the lipid-lowering efficacy of plant sterols/stanols was similar when the sterols were consumed as part of the diet and when they administered as a nutraceutical supplement (19), thus allowing for flexibility in the method of drug delivery.

Some evidence exists regarding the effect of sterol consumption on cardiovascular outcomes. Observational data suggest that high intake of plant sterols might be associated with MI prevention in men (22). A recent, large observational study indicated that natural phytosterol intake was associated with TC and LDL-C reduction particularly in men. However, this beneficial effect on lipid profile did not result in a reduction in the risk of CVD (23). One explanation for the LDL-C reduction failing to translate into a reduction in CVD is that sterols/stanols may reduce the absorption of carotenoids and fat-soluble vitamins (83). Such an effect would be expected to be associated with a higher incidence of CVD (84), however further investigations are needed to determine whether this effect occurs in vivo.

\section{Polyphenols}

Several studies have indicated that grape polyphenols may influence plasma lipid concentrations. Consumption of grape juice has been associated with elevated HDL-C (27). A study of the effect of polyphenol-rich grape extract supplement $(700 \mathrm{mg})$ on cardiovascular risk in healthy subjects and found that treatment was associated with a reduction in plasma TC and LDL-C concentrations (28). However, more extensive evidence in the form of a metaanalysis of nine randomized controlled trials (including 390 participants in total) did not find any effect of grape seed extract on LDL-C (29). While no effect was seen in this combined population, investigations of subpopulations and more sophisticated analysis of lipids has been carried out in other studies. One study found that plasma concentrations of large LDL-C and large LDL particles compared with placebo were decreased in obese subjects supplemented with grape powder for 3 weeks (30). However, the most atherogenic small LDL particles were not affected by treatment (85).

Despite, great enthusiasm with respect to the potential health benefits of resveratrol supplementation, a recent meta-analysis did not demonstrate any effect of this compound on plasma levels of TC, LDL-C, triglycerides or glucose. A small reduction in HDL-C concentrations was observed (41). Larger, well-designed trials are necessary to confirm these outcomes.

The results of studies investigating the effects of cocoa products on lipid profiles have been summarized in a metaanalysis of six randomized controlled trials (43). The results indicated that short-term cocoa consumption significantly lowered LDL-C by $5.87 \mathrm{mg} / \mathrm{dL}$, marginally lowered TC by $5.82 \mathrm{mg} / \mathrm{dL}$ without any evidence of an effect upon HDL-C concentrations. The effects appeared to be dosedependent and were observed in the subjects with elevated CVD risks but not in healthy participants (43). A variety of cocoa derivatives exist and do not all necessarily have similar biological effects. Dark chocolate (which contains cocoa butter and cocoa powder) consumption was compared to white chocolate (which contains cocoa butter but not powder) consumption and no difference between the groups was observed in terms of triglyceride, LDL-C and HDL-C in diabetic patients with hypertension (44). Previous studies have failed to observe benefits of cocoa polyphenols on blood lipids in subjects with hypertension stage 1 (45), overweight (46) and in patients with heart failure (47). A recent meta-analysis of 19 randomized controlled trials of varying designs with a total of 1,131 participants indicated that cocoa flavanols were associated with reductions in total triglycerides $(-0.10 \mathrm{mmol} / \mathrm{L})$ and increases in HDL-C $(0.06 \mathrm{mmol} / \mathrm{L})$ intake $(48)$. Recently published results from the Flaviola Health Study revealed that twicedaily ingestion of $450 \mathrm{mg}$ of cocoa flavanols for 1 month decreased TC by $0.20 \mathrm{mmol} / \mathrm{L}$ and LDL-C by $0.17 \mathrm{mmol} / \mathrm{L}$ whereas HDL-C increased by $0.10 \mathrm{mmol} / \mathrm{L}$ in a low risk, primary prevention population. The authors applied the Framingham Risk Score to their results and concluded that cocoa flavanols predicted a significant lowering of 10-year 
risk for CVD (49), however only well-designed (preferably randomized) studies can confirm whether this is the case.

Tea polyphenols may also exert lipid-lowering effects. A meta-analysis of 14 randomized controlled trials including 1,136 subjects in total found that the administration of green tea beverages or extracts resulted in significant reductions in serum TC and LDL-C concentrations, without altering HDL-C (56). Another meta-analysis of 20 randomized controlled trials appeared to confirm these results (57). In another study, both green and black tea and indicated that both reduced LDL-C whereas green tea also reduced TC (58). Similar properties of black tea were also confirmed by Zhao et al. (59). These observations highlight one of the difficulties of population research into nutraceuticals: small differences in the preparation of foods, which are not always captured in food-frequency questionnaires, can result in important differences in the composition of the foods and therefore can result in varying biological effects. Black tea consumption was shown to be more effective in lowering LDL-C in subjects with hypercholesterolemia and other markers of elevated cardiovascular risk (59). A recent randomized, placebocontrolled trial demonstrated reduced LDL-C and nonHDL-C after long-term supplementation (12 months) with green tea extract in healthy postmenopausal women (60).

Most studies to date have shown that plasma concentrations of HDL-C in humans are not affected by supplementation with grape products, cocoa and tea polyphenol. The exact mechanisms by green tea exert their LDL-C, lowering effects is not fully understood. Nevertheless, green tea is easily accessible, popular and safe and it may indirectly lead to lower morbidity and mortality rates due to CVD by improving hyperlipidemia outcomes.

Armolipid Plus is a food supplement combining natural ingredients containing red yeast rice, policosanol, berberine, folic acid, astaxanthin and coenzyme Q10. It has been demonstrated that supplementation with this nutraceutical exert reduction of TC $(-19.2 \%)$, LDL-C $(-17.4 \%)$ and triglycerides $(-16.3 \%)(68)$. A very recent meta-analysis of several randomized controlled trials revealed that this nutraceutical is safe, well tolerated and confirmed the beneficial effects upon lipid profile with reductions in plasma TC of $11-21 \%$ and reductions in LDL-C of $15-$ $31 \%(69)$.

\section{Spirulina}

Spirulina supplementation has been associated with beneficial alterations to blood lipid profiles $(70,71)$. Spirulina maxima, given orally ( $4.5 \mathrm{~g} / \mathrm{day}$, for 6 weeks), was associated with significant changes in TC and LDL-C concentrations (72). Furthermore, in a population of individuals with dyslipidemia, consumption of $1 \mathrm{~g}$ Spirulina per day for 12 weeks decreased mean levels of triglycerides, LDL-C, and TC without any apparent effect on plasma concentrations of HDL-C (73). A recent meta-analysis of seven randomized controlled trials with Spirulina appeared to confirm these findings (74). Further well-designed trials are required to clarify the mechanism of action of Spirulina supplementation in dyslipidemia and to determine its effects on cardiovascular outcomes.

Table $S 1$ summarizes the methodology and main findings from each study described in the paper.

\section{Hypertension}

Hypertension is an important modifiable risk factor for CVD (86). It has been shown that lowering blood pressure reduces CV risk by $20-25 \%$ for myocardial infarction, $35-40 \%$ for stroke and about $50 \%$ for heart failure (87). The evidence relating to antihypertensive effects of selected nutraceuticals is outlined below.

\section{Sterols/stanols}

In contrast to the well-studied effects of sterols and stanols upon lipids, there is a relative paucity of evidence relating to their effects upon blood pressure. Studies have failed to demonstrate antihypertensive effects of sterols/stanols despite continuing treatment for a year or more $(24,25)$. One recent study, which aimed to analyse the effect of plant stanol esters on arterial stiffness and endothelial function in adults also found no effect on measured blood pressure (26). Although the data are limited there appears to be no antihypertensive effects of these compounds in humans, neither is there any indication of adverse effects on blood pressure.

\section{Polyphenols}

It has been suggested that consumption of flavonoid-rich fruits and vegetables may lower blood pressure (88). Studies on the influence of polyphenols on blood pressure are very diverse and have included a great variety of polyphenolcontaining foods, including: grapes, berries, cocoa product, tea and other. Some studies have demonstrated a significant beneficial effect of grape-derived polyphenols on blood 
pressure $(31,32)$. On the other hand, there also numerous studies which did not identify such an association (33-35). The conflicting results probably result from the heterogeneity of study design, polyphenol source and population characteristics.

A recently published randomized controlled trial indicated that grape seed extract significantly reduced systolic blood pressure by $5.6 \%$ and diastolic blood pressure by $4.7 \%$ after 6 weeks of supplementation in subject with mildly elevated blood pressure. Moreover, the blood pressure lowering effects appeared to be dependent on baseline blood pressure, with the greatest reduction observed in subjects with higher baseline blood pressure (36).

The effects of resveratrol on blood pressure have also been studied. A meta-analysis of six randomized controlled trials (247 subjects), appeared to show a doseresponse relationship only higher doses ( $\geq 150 \mathrm{mg}$ day-1) of resveratrol significantly reduced systolic blood pressure while there were no significant effects on diastolic blood pressure (42). Another meta-analysis, which included data from ten randomized controlled trials indicated that resveratrol supplementation did not affect systolic and diastolic blood pressure (41). Differences in the baseline blood pressure of participants in the study may account for some of these discrepancies.

Cocoa flavanols are the most studied polyphenols in the clinical setting. A meta-analysis of 20 randomized controlled trials involving healthy participants indicated that consumption of polyphenol-rich cocoa products was associated with a reduction in blood pressure (50). A metaanalysis of 42 randomized controlled trials indicated that chocolate or cocoa was associated with reduced diastolic blood pressure and mean arterial pressure (51). Very recent clinical studies also confirm that cocoa flavanols exert a beneficial impact on blood pressure in patients with type 2 diabetes and hypertension (44) and in elderly subjects (52). Moreover, a recent study has demonstrated similar findings after cocoa ingestion in healthy individuals (53). This was associated with a dose-dependent improvement in flowmediated dilation, decreased endothelin-1 and pulse wave velocity. These findings warrant further investigation into the potential uses for cocoa in the prevention of CVD (53).

Conversely, another study found no evidence of an effect of daily intake of dark chocolate (49 g/day) on blood pressure or arterial stiffness in patients with mild hypertension (54). The Flaviola Health Study found that cocoa flavanol intake $(450 \mathrm{mg})$ for 1 month decreased systolic and diastolic blood pressure by 4.4 and $3.9 \mathrm{mmHg}$, pulse wave velocity by $0.4 \mathrm{~m} / \mathrm{s}$ and led to improved endothelial function in a healthy, primary prevention population, suggesting potential benefits of cocoa in maintain cardiovascular health (49).

Studies on tea polyphenols have also reported blood pressure lowering properties $(58,61)$. A meta-analysis of 10 trials (834 participants) demonstrated that a statistically significant reduction in systolic blood pressure was associated with diastolic blood pressure with black or green tea consumption (62). Furthermore, a recent meta-analysis on the effects of green tea intake on risk of CVD indicated that consumption of green tea is associated with reduced risk of myocardial infarction and stroke (63).

Several clinical studies have demonstrated an antihypertensive effect of pomegranate juice. A recent metaanalysis, which included the results of eight randomized controlled trials investigating the effects of pomegranate juice on blood pressure indicated that consumption of this polyphenol-rich juice significantly reduced both systolic and diastolic blood pressure (89). In addition, lipid-lowering, antioxidant and anti-atherosclerotic actions of pomegranate juice have been reported, making it a very attractive candidate as a nutraceutical with the potential to improve cardiovascular health (90).

The nutraceutical product, Armolipid Plus (containing red yeast rice, policosanol, berberine, folic acid, astaxanthin and coenzyme Q10) was found to be safe, well tolerated and effective in reducing mean 24-h systolic and 24-h pulse pressure in hypertensive and hypercholesterolemic subjects at low cardiovascular risk (68).

\section{Spirulina}

Some studies indicated that Spirulina maxima might exert an antihypertensive effect. Oral Spirulina supplementation resulted in systolic and diastolic blood pressure reduction in a small clinical trial (72). Conversely, no effect of Spirulina upon blood pressure was observed after consumption of $1 \mathrm{~g}$ Spirulina per day for 12 weeks in a Greek population (73). Administration of $2 \mathrm{~g}$ Hawaiian Spirulina for 3 months was associated with improved blood pressure and endothelial function in patients with hypertension. However, evidence relating to cardiovascular outcomes is lacking and further well-designed trials are required to clarify the clinical value of Spirulina supplementation in lowering blood pressure (75).

Table $S 1$ summarizes the methodology and main findings from each study described in the paper. 


\section{Diabetes mellitus}

Diabetes mellitus is a well-established risk factor for CVD. Diabetes mellitus type 2 is associated with high risk for developing cardiovascular complications (91). Moreover, patients with diabetes and hypertension have about twice the risk of cardiovascular events as nondiabetic patients with hypertension (92). It has been estimated that the global prevalence of diabetes mellitus will rise to 552 million by 2030 (93). Observational studies indicated that diet is one of the factors, which might prevent diabetes and its complications (94). The TOSCA-IT (the influence of dietary fat and carbohydrates proportions on plasma lipids, glucose control and low-grade inflammation in patients with type 2 diabetes) study demonstrated that a diet characterized by a higher intake of total polyphenols was associated with a better cardiovascular risk factors profile and a lower grade of subclinical inflammation in population with diabetes mellitus type 2 (95).

\section{Polyphenols}

Relatively few studies have evaluated the effects of grape polyphenol on hyperglycemia. Guilford et al. (96) indicated that regular red wine consumption is associated with a $30 \%$ risk reduction for type 2 diabetes. Consumption of grape seed polyphenols and red wine grape pomace flour have been associated with significant reductions in blood glucose $(37,97)$. Conversely another study found no effect of grape juice polyphenols in healthy individuals (38).

A randomized controlled trial revealed that supplementation of resveratrol for 3 months significantly improved the mean hemoglobin A1c, in patients with type 2 diabetes mellitus (39). A recent meta-analysis of 11 randomized controlled trials found that resveratrol reduced fasting glucose, insulin, glycated hemoglobin, and insulin resistance in subjects with type 2 diabetes, but not in those without diabetes (40). Conversely, a meta-analysis of 10 randomized controlled trials did not indicate a significant effect of resveratrol supplementation upon glucose level (41). The mechanism of action of resveratrol in the treatment of diabetes mellitus seems to be multifactorial; resveratrol may have antioxidant properties, increase AMPK activation, and increase internalization of glucose through modulating glucose transporter expression (98-100).

Results from studies evaluating influence of cocoa and tea polyphenols on glycemic markers are inconclusive. A metaanalysis of 42 randomized controlled trials indicated that chocolate or cocoa reduced fasting insulin concentrations, insulin after glucose challenge and improved insulin resistance. However, no effect upon fasting glucose or HbA1c was observed (51). Consumption of chocolate high in polyphenols was not associated with differences in fasting insulin in patients with diabetes mellitus type 2 and hypertension (44).

Another study found an independent inverse relationship between daily chocolate intake and concentrations of insulin, homeostatic model assessment of insulin resistance (HOMA-IR) and liver enzymes. Results of that study suggest that chocolate intake result in beneficial changes in liver enzymes and protect against insulin resistance (55). The results of a randomized controlled trial also support a role for cocoa flavanol intake in improving insulin resistance (48). The current data is insufficient to recommend chocolate and cocoa for glycemic control.

A meta-analysis of 17 trials comprising a total of 1,133 subjects revealed that green tea consumption was associated with significantly reduced fasting glucose and hemoglobin A1c (64). Furthermore, a meta-analysis of has also indicated changes in fasting glucose but glycated hemoglobin similar as fasting blood insulin and HOMA-IR did not change after green tea catechins administration (65). In a meta-analysis of 10 trials, which evaluated effect of green tea on populations at risk of diabetes mellitus type 2, green tea was not associated with reduced levels of fasting plasma glucose, fasting serum insulin, 2-h plasma glucose in the oral glucose tolerance test, hemoglobin A1c (HbA1c) and HOMA-IR (66).

Recent meta-analysis of 10 trials including 608 subjects which aimed to indicate the effect of green tea in patients with diabetes mellitus type 2 found that tea could alleviate the decrease of fasting blood insulin and reduced waist circumference only when the intervention was extended for longer than 8 weeks. No effects of tea on HOMAIR, fasting blood glucose, LDL-C, HDL-C, body mass index, systolic blood pressure, diastolic blood pressure, triglycerides and fasting cholesterol were observed (67). These mixed results mean that it is unclear whether green tea has anti-diabetic effects.

Polyphenols might influence glucose homeostasis by several mechanisms, by inhibiting carbohydrate digestion and glucose absorption in the intestine, protecting pancreatic $\beta$-cells, reducing glucose release from liver and activating insulin receptors and glucose uptake in insulinsensitive tissues (101). 


\section{Spirulina}

Spirulina might be beneficial in controlling blood glucose level in subject with diabetes mellitus type 2 . Supplementation of $2 \mathrm{~g} /$ day for 2 months resulted in reduced fasting blood glucose, postprandial blood glucose levels and HbA1c (70). Conversely oral supplementation of Spirulina in a separate trial ( $4.5 \mathrm{~g} /$ day, for 6 weeks) did not result in changes in the markers of glucose metabolism (72). Further trials are required to clarify the clinical value of Spirulina supplementation in treatment of diabetes.

Table S1 summarizes the methodology and main findings from each study described in the paper.

\section{Conclusions}

Dyslipidemia, hypertension and diabetes are major modifiable risk factors for CVD. Current medical treatments for the management of diabetes and dyslipidemia in some especially high risk patients are insufficient and current evidence suggests that the application of nutraceuticals may have the potential to increase the effectiveness of therapy (as well as to reduce the residual risk). Many of the nutraceuticals investigated for the prevention and treatment of CVD are well tolerated in patients. However, there is often insufficient data available with respect to long-term safety and effectiveness against clinical outcomes such as myocardial infarction and mortality. Further clinical research should be conducted to identify nutraceuticals with the best clinical and cost-effectiveness in the prevention and treatment of CVD (102-105).

\section{Acknowledgements}

None.

\section{Footnote}

Conflicts of Interest: The authors have no conflicts of interest to declare.

\section{References}

1. Roth GA, Huffman MD, Moran AE, et al. Global and regional patterns in cardiovascular mortality from 1990 to 2013. Circulation 2015;132:1667-78.

2. Voelter-Mahlknecht S. Epigenetic associations in relation to cardiovascular prevention and therapeutics. Clin
Epigenetics 2016;8:4.

3. Piepoli MF, Hoes AW, Agewall S, et al. 2016 European Guidelines on cardiovascular disease prevention in clinical practice. Eur Heart J 2016;37: 2315-81.

4. O'Keeffe C, Kabir Z, O'Flaherty M, et al. Modelling the impact of specific food policy options on coronary heart disease and stroke deaths in Ireland. BMJ Open 2013;3: e002837.

5. Alissa EM, Ferns GA. Functional foods and nutraceuticals in the primary prevention of cardiovascular diseases. J Nutr Metab 2012;2012:569486.

6. Tresserra-Rimbau A, Rimm EB, Medina-Remón A, et al. Inverse association between habitual polyphenol intake and incidence of cardiovascular events in the PREDIMED study. Nutr Metab Cardiovasc Dis 2014; 24:639-47.

7. DeFelice SL. The nutraceutical revolution: its impact on food industry R\&D. Trends in Food Science and Technology 1995;2:59-61.

8. Moreau RA, Whitaker BD, Hicks KB. Phytosterols, Phytostanols, and Their Conjugates in Foods: Structural Diversity, Quantitative Analysis, and Health-Promoting Uses. Prog Lipid Res 2002;41:457-500.

9. Pandey KB, Rizvi SI. Plant polyphenols as dietary antioxidants in human health and disease. Oxid Med Cell Longev 2009;2:270-8.

10. Spácil Z, Novakova L, Solich P. Analysis of phenolic compounds by high performance liquid chromatography and ultra-performance liquid chromatography. Talanta 2008;76:189-99.

11. Burns J, Yokota T, Ashihara H, et al. Plant foods and herbal sources of resveratrol. J Agric Food Chem 2002;50: 3337-40.

12. Andres-Lacueva C, Monagas M, Khan N, et al. Flavanol and flavonol contents of cocoa powder products: Influence of the manufacturing process. J Agric Food Chem 2008;56:3111-7.

13. Hammerstone JF, Lazarus SA, Mitchell AE, et al. Identification of procyanidins in cocoa (Theobroma cacao) and chocolate using high-performance liquid chromatography/mass spectrometry. J Agric Food Chem 1999;47:490-6.

14. Lee KW, Kim YJ, Lee HJ, et al. Cocoa has more phenolic phytochemicals and a higher antioxidant capacity than teas and red wine. J Agric Food Chem 2003;51:7292-5.

15. Khan N, Mukhtar H. Tea polyphenols for health promotion. Life Sci 2007;81:519-33.

16. Balentine DA, Wiseman SA, Bouwens LC. The chemistry of tea flavonoids. Crit Rev Food Sci Nutr 1997;37: 693-704.

17. Khan Z, Bhadouria P, Bisen P. Nutritional and therapeutic 
potential of Spirulina. Curr Pharm Biotechnol 2005; 6:373-9.

18. Sarubin-Fragakis A. The health professional's guide to popular dietary supplements. Am J Clin Nutr 2003;78:808

19. Amir Shaghaghi M, Abumweis SS, Jones PJ. Cholesterollowering efficacy of plant sterols/stanols provided in capsule and tablet formats: results of a systematic review and meta-analysis. J Acad Nutr Diet 2013;113:1494-503.

20. Ras RT, Geleijnse JM, Trautwein EA. LDL cholesterollowering effect of plant sterols and stanols across different dose ranges: A meta-analysis of randomised controlled studies. Br J Nutr 2014;112:214-9.

21. Han S, Jiao J, Xu J, et al. Effects of plant stanol or sterolenriched diets on lipid profiles in patients treated with statins: systematic review and meta-analysis. Sci Rep 2016;6:31337.

22. Klingberg S, Ellegard L, Johansson I, et al. Dietary intake of naturally occurring plant sterols is related to a lower risk of a first myocardial infarction in men but not in women in northern Sweden. J Nutr 2013;143:1630-5.

23. Ras RT, van der Schouw YT, Trautwein EA, et al. Intake of phytosterols from natural sources and risk of cardiovascular disease in the European prospective investigation into cancer and nutrition- the Netherlands (EPIC-NL) population. Eur J Prev Cardiol 2015;22:1067-75.

24. Gylling H, Hallikainen M, Raitakari OT, et al. Longterm consumption of plant stanol and sterol esters, vascular function and genetic regulation. Br J Nutr 2009;101:1688-95.

25. Raitakari OT, Salo P, Ahotupa M. Carotid artery compliance in users of plant stanol ester margarine. Eur J Clin Nutr 2008;62:218-24.

26. Gylling H, Halonen J, Lindholm H, et al. The effects of plant stanol ester consumption on arterial stiffness and endothelial function in adults: a randomised controlled clinical trial. BMC Cardiovasc Disord 2013;13:50.

27. Khadem-Ansari MH, Rasmi Y, Ramezani F. Effects of red grape juice consumption on high density lipoproteincholesterol, apolipoprotein AI, apolipoprotein B and homocysteine in healthy human volunteers. Open Biochem J 2010;4:96-9.

28. Yubero N, Sanz-Buenhombre M, Guadarrama A, et al. LDL cholesterol-lowering effects of grape extract used as a dietary supplement on healthy volunteers. Int J Food Sci Nutr 2013;64:400-6.

29. Feringa HH, Laskey DA, Dickson JE, et al. The effect of grape seed extract on cardiovascular risk markers: a meta- analysis of randomized controlled trials. J Am Diet Assoc 2011;111:1173-81.

30. Zunino SJ, Peerson JM, Freytag TL, et al. Dietary grape powder increases IL- $1 \beta$ and IL- 6 production by lipopolysaccharide-activated monocytes and reduces plasma concentrations of large LDL and large LDLcholesterol particles in obese humans. Br J Nutr 2014;112:369-80.

31. Barona J, Aristizabal JC, Blesso CN, et al. Grape polyphenols reduce blood pressure and increase flowmediated vasodilation in men with metabolic syndrome. J Nutr 2012;142:1626-32.

32. Draijer R, de Graaf $Y$, Slettenaar $M$, et al. Consumption of a polyphenol-rich grape-wine extract lowers ambulatory blood pressure in mildly hypertensive subjects. Nutrients 2015;7:3138-53.

33. Vaisman N, Niv E. Daily consumption of red grape cell powder in a dietary dose improves cardiovascular parameters: a double blind, placebo-controlled, randomized study. Int J Food Sci Nutr 2015;66:342-9.

34. Hodgson JM, Croft KD, Woodman RJ, et al. Effects of vitamin $\mathrm{E}$, vitamin $\mathrm{C}$ and polyphenols on the rate of blood pressure variation: results of two randomized controlled trials. Br J Nutr 2014;112:1551-61.

35. Díaz-Rubio ME, Perez-Jimenez J, Martinez-Bartolome MA, et al. Regular consumption of an antioxidant-rich juice improves oxidative status and causes metabolome changes in healthy adults. Plant Foods Hum Nutr 2015;70:9-14.

36. Park E, Edirisinghe I, Choy YY, et al. Effects of grape seed extract beverage on blood pressure and metabolic indices in individuals with pre-hypertension: a randomized, double-blinded, two-arm, parallel, placebo-controlled trial. Br J Nutr 2016;115:226-38.

37. Urquiaga I, D'Acuña S, Pérez D, et al. Wine grape pomace flour improves blood pressure, fasting glucose and protein damage in humans: a randomized controlled trial. Biol Res 2015;48:49.

38. Toaldo IM, Cruz FA, da Silva EL, et al. Acute consumption of organic and conventional tropical grape juices (Vitis labrusca L.) increases antioxidants in plasma and erythrocytes, but not glucose and uric acid levels, in healthy individuals. Nutr Res 2016;36:808-17.

39. Bhatt JK, Thomas S, Nanjan MJ. Resveratrol supplementation improves glycemic control in type 2 diabetes mellitus. Nutr Res 2012;32:537-41.

40. Liu K, Zhou R, Wang B, et al. Effect of resveratrol on glucose control and insulin sensitivity: a meta-analysis 
of 11 randomized controlled trials. Am J Clin Nutr 2014;99:1510-9.

41. Sahebkar A, Serban C, Ursoniu S, et al. Lipid and Blood Pressure Meta-analysis Collaboration Group. Lack of efficacy of resveratrol on C-reactive protein and selected cardiovascular risk factors--Results from a systematic review and meta-analysis of randomized controlled trials. Int J Cardiol 2015;189:47-55.

42. Liu Y, Ma W, Zhang P, et al. Effect of resveratrol on blood pressure: a meta-analysis of randomized controlled trials. Clin Nutr 2015;34:27-34.

43. Jia L, Liu X, Bai YY, et al. Short-term effect of cocoa product consumption on lipid profile: a meta-analysis of randomized controlled trials. Am J Clin Nutr 2010;92:218-25.

44. Rostami A, Khalili M, Haghighat N, et al. High-cocoa polyphenol-rich chocolate improves blood pressure in patients with diabetes and hypertension. ARYA Atheroscler 2015;11:21-9.

45. Nogueira Lde P, Knibel MP, Torres MR, et al. Consumption of High-Polyphenol Dark Chocolate Improves Endothelial Function in Individuals with Stage 1 Hypertension and Excess Body Weight. Int J Hypertens 2012;2012:147321.

46. West SG, McIntyre MD, Piotrowski MJ, et al. Effects of dark chocolate and cocoa consumption on endothelial function and arterial stiffness in overweight adults. Br J Nutr 2014;111:653-61.

47. Flammer AJ, Sudano I, Wolfrum M, et al. Cardiovascular effects of flavanol-rich chocolate in patients with heart failure. Eur. Heart J 2012;33:2172-80.

48. Lin X, Zhang I, Li A, et al. Cocoa Flavanol Intake and Biomarkers for Cardiometabolic Health: A Systematic Review and Meta-Analysis of Randomized Controlled Trials. J Nutr 2016;146:2325-33.

49. Sansone R, Rodriguez-Mateos A, Heuel J, et al. Cocoa flavanol intake improves endothelial function and Framingham Risk Score in healthy men and women: a randomized, controlled, double-masked trial: the Flaviola Health Study. Br J Nutr 2015;114:1246-55.

50. Ried K, Sullivan TR, Fakler P, et al. Effect of cocoa on blood pressure. Cochrane Database Syst Rev 2012;15:CD008893.

51. Hooper L, Kay C, Abdelhamid A, et al. Effects of chocolate, cocoa, and flavan-3-ols on cardiovascular health: A systematic review and meta-analysis of randomized trials. Am J Clin Nutr 2012;95:740-51.

52. Mastroiacovo D, Kwik-Uribe C, Grassi D, et al. Cocoa flavanol consumption improves cognitive function, blood pressure control, and metabolic profile in elderly subjects: the Cocoa, Cognition, and Aging (CoCoA) Study - a randomized controlled trial. Am J Clin Nutr 2015;101:538-48.

53. Grassi D, Desideri G, Necozione S, et al. Cocoa consumption dose-dependently improves flow-mediated dilation and arterial stiffness decreasing blood pressure in healthy individuals. J Hypertens 2015;33:294-303.

54. Koli R, Köhler K, Tonteri E, et al. Dark chocolate and reduced snack consumption in mildly hypertensive adults: an intervention study. Nutr J 2015;14:84.

55. Alkerwi A, Sauvageot N, Crichton GE, et al. Daily chocolate consumption is inversely associated with insulin resistance and liver enzymes in the Observation of Cardiovascular Risk Factors in Luxembourg study. Br J Nutr 2016;115:1661-8.

56. Zheng XX, Xu YL, Li SH, et al. Green tea intake lowers fasting serum total and LDL cholesterol in adults: a metaanalysis of 14 randomized controlled trials. Am J Clin Nutr 2011;94:601-10.

57. Kim A, Chiu A, Barone MK, et al. Green tea catechins decrease total and low-density lipoprotein cholesterol: a systematic review and meta-analysis. J Am Diet Assoc 2011;111:1720-9.

58. Hartley L, Flowers N, Holmes J, et al. Green and black tea for the primary prevention of cardiovascular disease. Cochrane Database Syst Rev 2013;18:CD009934.

59. Zhao Y, Asimi S, Wu K, et al. Black tea consumption and serum cholesterol concentration: Systematic review and meta-analysis of randomized controlled trials. Clin Nutr 2015;34:612-9.

60. Samavat H, Newman AR, Wang R, et al. Effects of green tea catechin extract on serum lipids in postmenopausal women: a randomized, placebo-controlled clinical trial. Am J Clin Nutr 2016;104:1671-82.

61. Khalesi S, Sun J, Buys N, et al. Green tea catechins and blood pressure: a systematic review and meta-analysis of randomised controlled trials. Eur J Nutr 2014;53:1299-311.

62. Yarmolinsky J, Gon G, Edwards P. Effect of tea on blood pressure for secondary prevention of cardiovascular disease: a systematic review and meta-analysis of randomized controlled trials. Nutr Rev 2015;73:236-46.

63. Pang J, Zhang Z, Zheng T, et al. Green tea consumption and risk of cardiovascular and ischemic related diseases: A meta-analysis. Int J Cardiol 2016;202:967-74.

64. Liu K, Zhou R, Wang B, et al. Effect of green tea on glucose control and insulin sensitivity: a meta-analysis 
of 17 randomized controlled trials. Am J Clin Nutr 2013;98:340-8.

65. Zheng XX, Xu YL, Li SH, et al. Effects of green tea catechins with or without caffeine on glycemic control in adults: a meta-analysis of randomized controlled trials. Am J Clin Nutr 2013;97:750-62.

66. Wang X, Tian J, Jiang J, et al. Effects of green tea or green tea extract on insulin sensitivity and glycaemic control in populations at risk of type 2 diabetes mellitus: a systematic review and meta-analysis of randomized controlled trials. J Hum Nutr Diet 2014;27:501-12.

67. Li Y, Wang C, Huai Q, et al. Effects of tea or tea extract on metabolic profiles in patients with type 2 diabetes mellitus: a meta-analysis of ten randomized controlled trials. Diabetes Metab Res Rev 2016;32:2-10.

68. Mazza A, Lenti S, Schiavon L, et al. Nutraceuticals for Serum Lipid and Blood Pressure Control in Hypertensive and Hypercholesterolemic Subjects at Low Cardiovascular Risk. Adv Ther 2015;32:680-90.

69. Barrios V, Escobar C, Cicero AF, et al. A nutraceutical approach (Armolipid Plus) to reduce total and LDL cholesterol in individuals with mild to moderate dyslipidemia: Review of the clinical evidence. Atheroscler Suppl 2017;24:1-15.

70. Parikh P, Mani U, Iyer U. Role of Spirulina in the Control of Glycemia and Lipidemia in Type 2 Diabetes Mellitus. J Med Food 2001;4:193-9.

71. Lee EH, Park JE, Choi YJ, et al. A randomized study to establish the effects of spirulina in type 2 diabetes mellitus patients. Nutr Res Pract 2008;2:295-300.

72. Torres-Duran PV, Ferreira-Hermosillo A, JuarezOropeza MA. Antihyperlipemic and antihypertensive effects of Spirulina maxima in an open sample of Mexican population: a preliminary report. Lipids Health Dis 2007;6:33.

73. Mazokopakis EE, Starakis IK, Papadomanolaki MG, et al. The hypolipidaemic effects of Spirulina (Arthrospira platensis) supplementation in a Cretan population: a prospective study. J Sci Food Agric 2014;94:432-7.

74. Serban MC, Sahebkar A, Dragan S, et al. A systematic review and meta-analysis of the impact of Spirulina supplementation on plasma lipid concentrations. Clin Nutr 2016;35:842-51.

75. Miczke A, Szulińska M, Hansdorfer-Korzon R, et al. Effects of spirulina consumption on body weight, blood pressure, and endothelial function in overweight hypertensive Caucasians: a double-blind, placebocontrolled, randomized trial. Eur Rev Med Pharmacol Sci
2016;20:150-6.

76. Reiner Z, Catapano AL, De Backer G, et al. ESC/EAS Guidelines for the Management of Dyslipidaemias. The Task Force for the Management of Dyslipidaemias of the European Society of Cardiology (ESC) and European Atherosclerosis Society (EAS). Developed with the special contribution of the European Association for Cardiovascular Prevention \& Rehabilitation (EACPR). Europ Heart J 2011;32:1769-818.

77. Silverman MG, Ference BA, Im K, et al. Association Between Lowering LDL-C and Cardiovascular Risk Reduction Among Different Therapeutic Interventions: A Systematic Review and Meta-analysis. JAMA 2016;316: 1289-97.

78. Sahebkar A, Serban MC, Gluba-Brzózka A, et al. Lipidmodifying effects of nutraceuticals: An evidence-based approach. Nutrition 2016;32:1179-92.

79. Andersson SW, Skinner J, Ellegard L, et al. Intake of dietary plant sterols is inversely related to serum cholesterol concentration in men and women in the EPIC Norfolk population: A cross-sectional study. Eur J Clin Nutr 2004;58:1378-85.

80. Wang P, Chen YM, He LP, et al. Association of natural intake of dietary plant sterols with carotid intima-media thickness and blood lipids in Chinese adults: A crosssection study. PLoS One 2012;7:e32736.

81. Gylling H, Plat J, Turley S, et al. for the European atherosclerosis society consensus panel on phytosterols. Plant sterols and plant stanols in the management of dyslipidaemia and prevention of cardiovascular disease. Atherosclerosis 2014;232:346-60.

82. de Jong A, Plat J, Mensink RP. Metabolic effects of plant sterols and stanols. J Nutr Biochem 2003;14;362-9.

83. Gylling H, Hallikainen M, Nissinen MJ, et al. The effect of a very high daily plant stanol ester intake on serum lipids, carotenoids, and fat soluble vitamins. Clin Nutr 2010;29:112-8.

84. Voutilainen S, Nurmi T, Mursu J, et al. Carotenoids and cardiovascular health. Am J Clin Nutr 2006;83:1265-71.

85. Koba S, Yokota Y, Hirano T, et al. Small LDL-cholesterol is superior to LDL-cholesterol for determining severe coronary atherosclerosis. J Atheroscler Thromb 2008; 15:250-60.

86. Papadogiannis DE, Protogerou AD. Blood pressure variability: A confounder and a cardiovascular risk factor. Hypertens Res 2011;34:162-3.

87. Antonakoudis G, Poulimenos L, Kifnidis K, et al. Blood pressure control and cardiovascular risk reduction. 
Hippokratia 2007;11:114-9.

88. Wang X, Ouyang Y, Liu J, et al. Fruit and vegetable consumption and mortality from all causes, cardiovascular disease, and cancer: systematic review and dose-response meta-analysis of prospective cohort studies. BMJ 2014;349:g4490.

89. Sahebkar A, Ferri C, Giorgini P, et al. Effects of pomegranate juice on blood pressure: A systematic review and meta-analysis of randomized controlled trials. Pharmacol Res 2017;115:149-61.

90. Asgary S, Keshvari M, Sahebkar A, et al. Pomegranate consumption and blood pressure: A review. Curr Pharm Des 2016. [Epub ahead of print].

91. Gu K, Cowie CC, Harris MI. Diabetes and decline in heart disease mortality in US adults. JAMA 1999;281:1291-7.

92. Arauz-Pacheco C, Parrott MA, Raskin P. The treatment of hypertension in adult patients with diabetes. Diabetes Care 2002;25:134-47.

93. Whiting DR, Guariguata L, Weil C, et al. IDF diabetes atlas: global estimates of the prevalence of diabetes for 2011 and 2030. Diabetes Res Clin Pract 2011;94:311-21.

94. Bosch J, Yusuf S, Gerstein HC, et al. Effect of ramipril on the incidence of diabetes. N Engl J Med 2006;355:1551-62.

95. Vitale M, Vaccaro O, Masulli M, et al. Polyphenol intake and cardiovascular risk factors in a population with type 2 diabetes: The TOSCA.IT study. Clin Nut 2016. [Epub ahead of print].

96. Guilford JM, Pezzuto JM. Wine and health: A review. Am J Enol Vitic 2011;62:471-86.

97. Akaberi M, Hosseinzadeh H. Grapes (Vitis vinifera) as a Potential Candidate for the Therapy of the Metabolic

Cite this article as: Sosnowska B, Penson P, Banach M. The role of nutraceuticals in the prevention of cardiovascular disease. Cardiovasc Diagn Ther 2017;7(Suppl 1):S21-S31. doi: 10.21037/cdt.2017.03.20
Syndrome. Phytother Res 2016;30:540-56.

98. Yao L, Wan J, Li H, et al. Resveratrol relieves gestational diabetes mellitus in mice through activating AMPK. Reprod Biol Endocrinol 2015;13:118.

99. Gencoglu H, Tuzcu M, Hayirli A, et al. Protective effects of resveratrol against streptozotocin-induced diabetes in rats by modulation of visfatin/sirtuin-1 pathway and glucose transporters. Int J Food Sci Nutr 2015;66:314-20.

100. Goh KP, Lee HY, Lau DP, et al. Effects of resveratrol in patients with type 2 diabetes mellitus on skeletal muscle SIRT1 expression and energy expenditure. Int J Sport Nutr Exerc Metab 2014;24:2-13.

101. Hanhineva K, Torronen R, Bondia-Pons I, et al. Impact of dietary polyphenols on carbohydrate metabolism. Int J Mol Sci 2010;11:1365-402.

102. Sahebkar A, Serban C, Ursoniu S, et al. Effect of garlic on plasma lipoprotein(a) concentrations: A systematic review and meta-analysis of randomized controlled clinical trials. Nutrition 2016;32:33-40.

103. Serban C, Sahebkar A, Antal D, et al. Effects of supplementation with green tea catechins on plasma C-reactive protein concentrations: A systematic review and meta-analysis of randomized controlled trials. Nutrition 2015;31:1061-71.

104. Banach M, Aronow WS, Serban C, et al. Lipids, blood pressure and kidney update 2014. Pharmacol Res 2015;9596:111-25.

105. Ursoniu S, Sahebkar A, Serban MC, et al. Lipid profile and glucose changes after supplementation with astaxanthin: a systematic review and meta-analysis of randomized controlled trials. Arch Med Sci 2015;11:253-66. 


\begin{tabular}{|c|c|c|}
\hline Peterence & 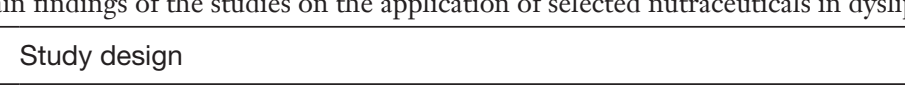 & Subjects characteristic \\
\hline Serolis stano & 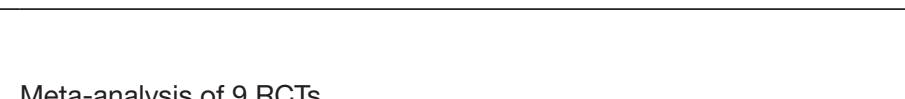 & \\
\hline (20) & Metazanalysis of of 124 RCT & $\begin{array}{ll}20502 \text { particicanis } \\
624 \text { patipants }\end{array}$ \\
\hline (21) & Meta-analysis of 15 RCTs & 500 patients treated with statins \\
\hline (22) & Nested case-referent study & 1,005 individuals (219 women, 786 men) \\
\hline (23) & Obserational study & 35,597 Dutch men and women \\
\hline $\begin{array}{l}(24) \\
(25)\end{array}$ & 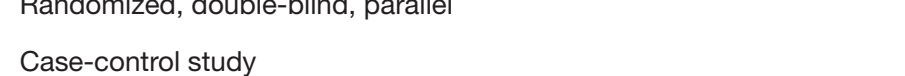 & 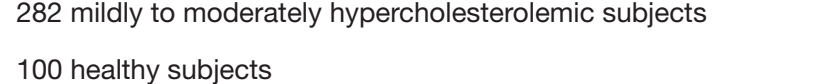 \\
\hline (26) & Randomized, controlled, double-bind, parallet trial & 92 asymptomatic subjects \\
\hline \multicolumn{3}{|c|}{ 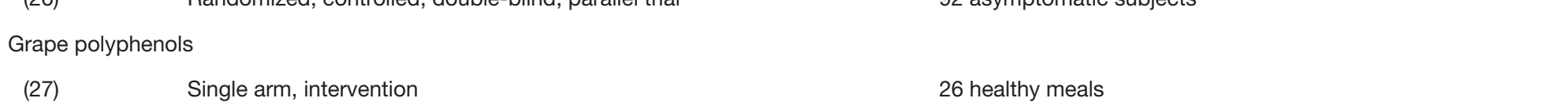 } \\
\hline (28) & 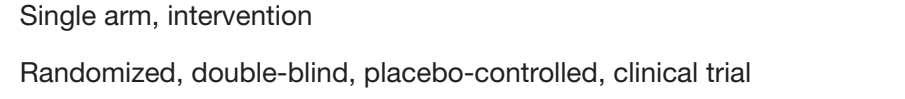 & $\begin{array}{l}20 \text { h hantry mans } \\
60 \text { heathy subjects }\end{array}$ \\
\hline (29) & Meta-analysis of 9 RCT & 390 participants \\
\hline (30) & 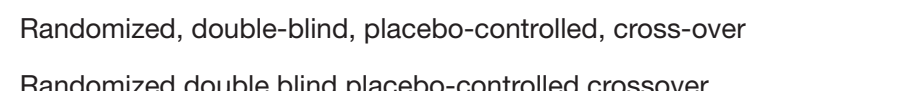 & 24 obese subjects \\
\hline (32) & 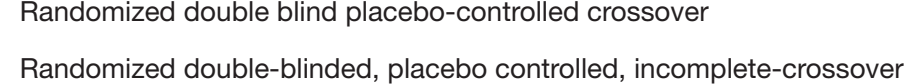 & $\begin{array}{l}2 \text { males with metabolic } 4 \text { mydrome } \\
6 \text { mases milly hypertensive }\end{array}$ \\
\hline (33) & Randomized, double-bind, placebo-controlled, parallel & 50 pre- and mild-hypertension subjects \\
\hline (34) & Randonized, double-bind, pacecb-conontroled trial & 69 hypertensive individuals \\
\hline (35) & 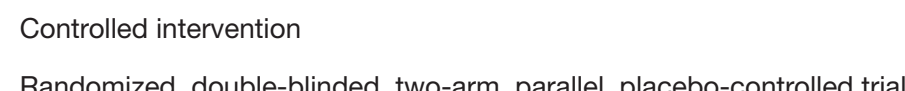 & 28 heathy young acults \\
\hline (37) & 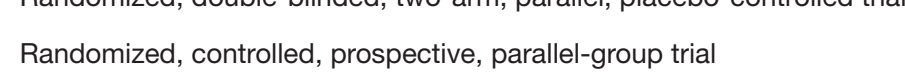 & 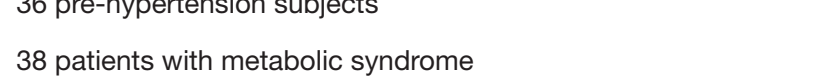 \\
\hline (38) & Randomirized, controlede, crossover, interenention trial & 24 heartyry indwoudals \\
\hline (39) & Randonized, prospective, open-1abel, controlede trial & 62 patients with Trom \\
\hline (40) & Metatanalysis of 1 RCTIs & 388 sujjects with and without T2DM \\
\hline & Metatanalysis of 10 BCTs & 575 participants \\
\hline \multicolumn{3}{|c|}{ Meta-analysis of 6 RCTs } \\
\hline (43) & Metaranalysis of 8 RCTs & 215 participants \\
\hline (44) & Randomized, placeso-controlled, double-blina study & T2DM patients with hypertension \\
\hline (45) & Intevention clinical tral & 22 subjects with hypertension stage 1 \\
\hline 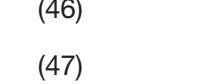 & Randomized, placeco- controlled, cross- vore study & 30 overwight adults \\
\hline (48) & Randomized, double-bilind, placecob-controlled trial & 22 patients with stable CHF (NYHA Z II) \\
\hline (49) & $\begin{array}{l}\text { Metatanalysis of } 19 \text { RCCls } \\
\text { Randomize, controled, dout }\end{array}$ & 1,131 participantits \\
\hline & Meta-analysis of $20 \mathrm{RCTS}$ & 856 mainly heathy participants \\
\hline & Meta-analysis of 42 RCTs & 1,297 participants \\
\hline (52) & 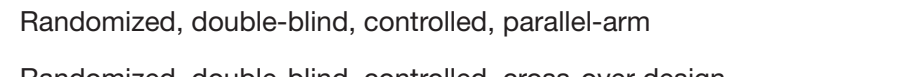 & go edderly subijects \\
\hline (5) & 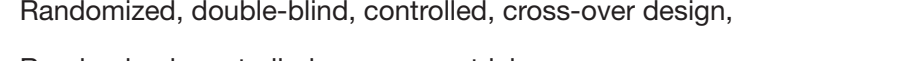 & 20 healthy individalas \\
\hline (55) & 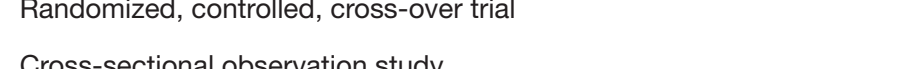 & 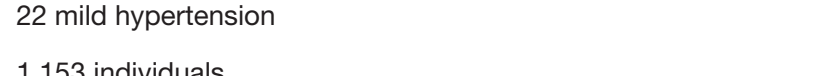 \\
\hline \multicolumn{3}{|c|}{ 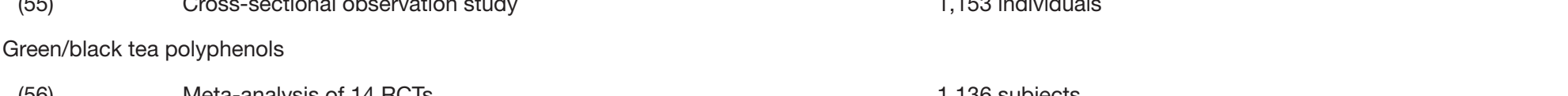 } \\
\hline (56) & Meta-analysis of 14 RCTs & 1,136 subjects \\
\hline & Metatanalysis of 20 ATCS & 1.4115 participants \\
\hline (58) & Metazanalysis of 1 1 RCTs & 821 participants \\
\hline (59) & Meta-analysis of 10 BCTs & 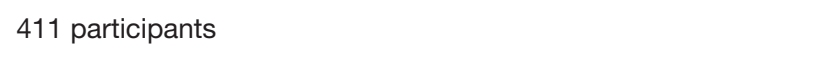 \\
\hline (60) & 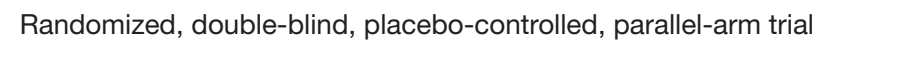 & 936 heathy postmenopoasusal women \\
\hline (61) & Meta-analysis of 13 RCTs & 947 paticicantis \\
\hline $\begin{array}{l}(62) \\
(63)\end{array}$ & 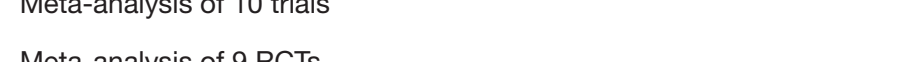 & 834 participants \\
\hline (64) & (1) & 259,267 individuals \\
\hline & 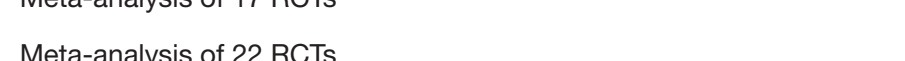 & 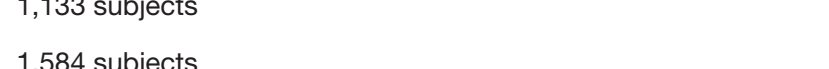 \\
\hline (66) & Meta-analysis of 7 RCTs & 510 sujects a t isk of T2DM \\
\hline & Meta-analysis of 10 trials & 608 subjects \\
\hline \\
\hline (69) & Meta-analysis of 13 RCTs & Mostly patients with mild to moderate dysipipidemia \\
\hline \multicolumn{3}{|l|}{ Spirulina } \\
\hline 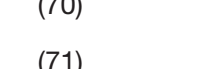 & 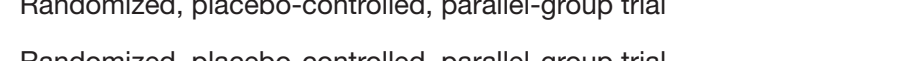 & 25 T200M subjects \\
\hline (72) & 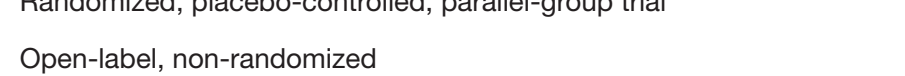 & 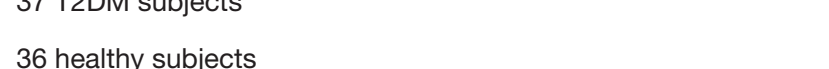 \\
\hline (73) & Open-1abel, non-randomized & 52 dyslipidemic subjec \\
\hline (74) & Meta-analysis of 7 RCTs & 522 participants \\
\hline
\end{tabular}
Intevention

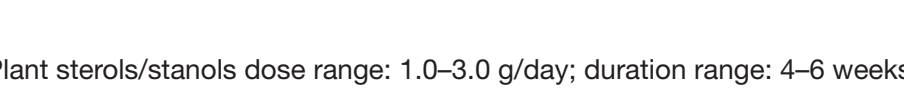

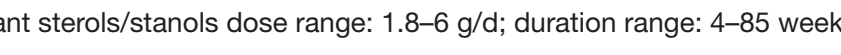

LDL-C reduction by 12 mgdol

(a)

Red grape juice $150 \mathrm{mLt}$ twice per day for 4 weels

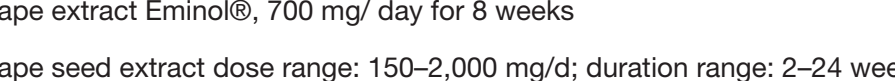

TC, LDLC, non-HDL-C declined by $6.6 \% \%$, $10.2 \%$, and $10.6 \%$, redo

ape powder, 46 g twice per day tor 3 weeks

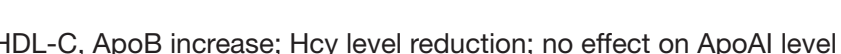

SPP (-1.54 mmHg), heart rate $-(-1.42$ b pom) reduction; no effect on DBP, lipid levels, CRP

Readuction of large LDL-Cand large LDL particles

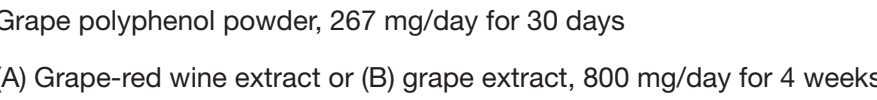

Red graee cell Dowder, 200 or 400 ma for 12 weeks

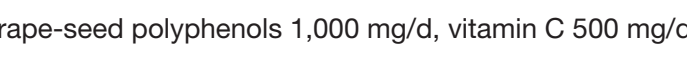

Grape and pomegeranatej jice, $200 \mathrm{~m} \amalg$ Lay for 8 -wewe

Red wine grape pomace flour, 20 grday for 16 weets

Tropical grape juce Nitis abusscal., $400 \mathrm{~m}$ L Lor 14 days

Resueratato, $250 \mathrm{mg} / \mathrm{d}$ for 3 months

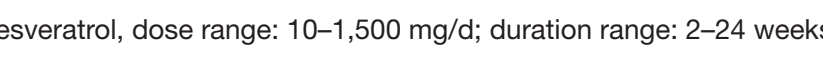

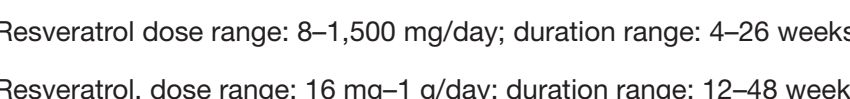

Coccap products dose range: 88-963 mglday; duration range: 2-18 weeks

Dark chocolate, 25 /day for 8 weeks

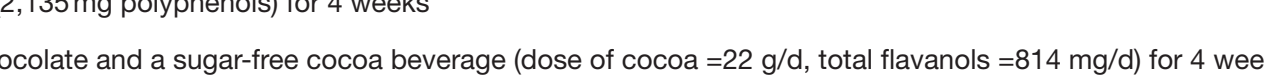

Flavanor-ich chococolate 40 g/d for 4 weeks

cocoa tlavanols, $450 \mathrm{mg}$ twice a day tor 4 weers

s per day, duration range: 2-18 weeks

Cocoa flavanols, 993,520 or $48 \mathrm{mg}$ for 8 weeks

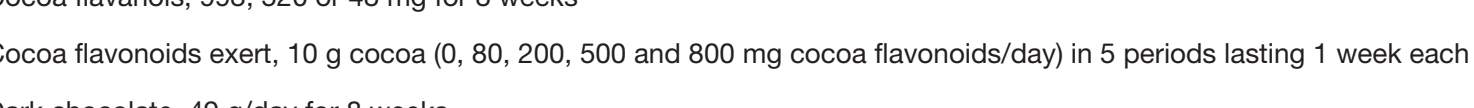

Dark choocolate, 49 g/day for 8 we

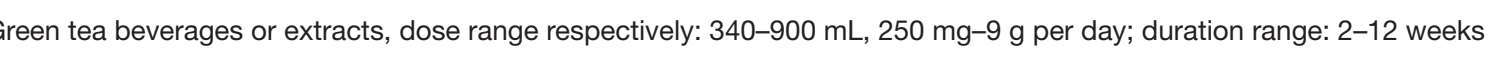

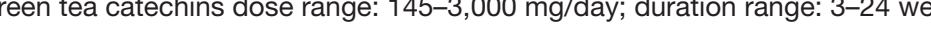

(a)

Green tea extract, $1315 \mathrm{mg}$ catecchins per day, duration: 12 monts

Green tea extract or beverages, catechins $100-1,500 \mathrm{mg}$, duration range: $-3-16$ weeks

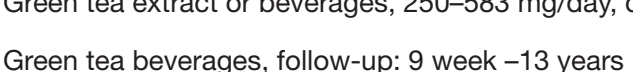

(a)

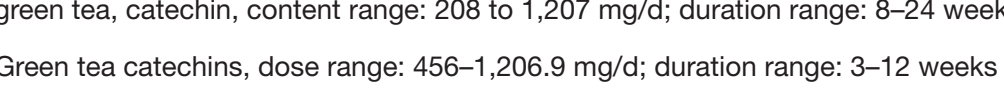

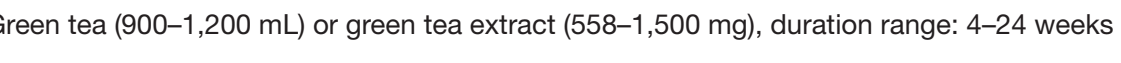

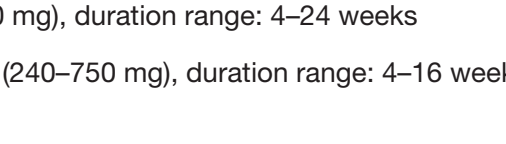

molipid plus, 1 tablet tor months

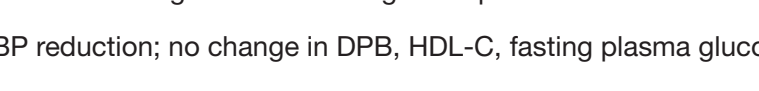

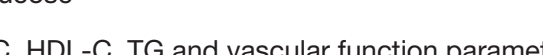

no eftect on SBP. FWD

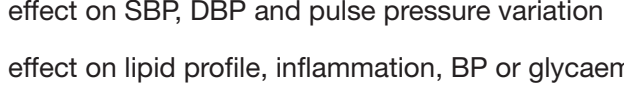

bood pressure, gyvearivia and postpprandial insulin

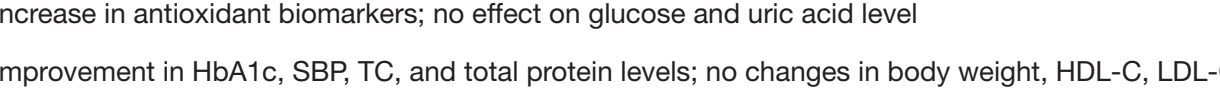

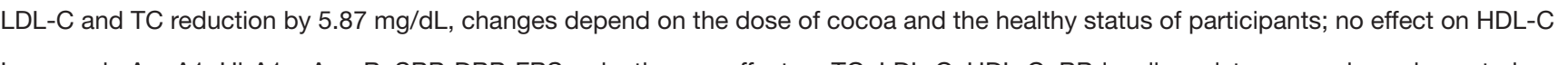

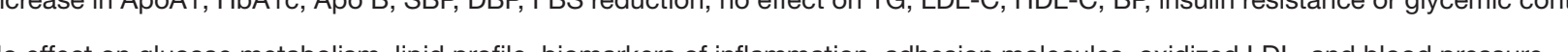

Wease in

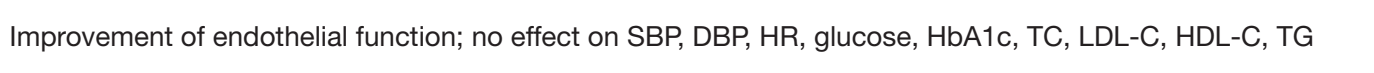

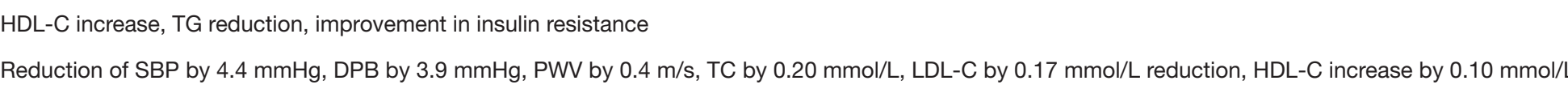

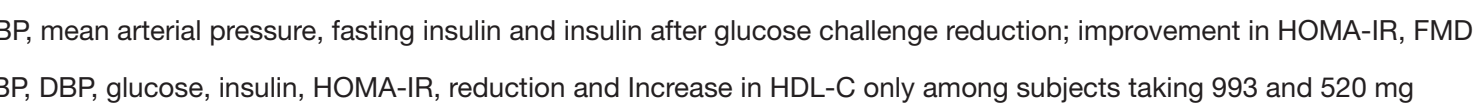

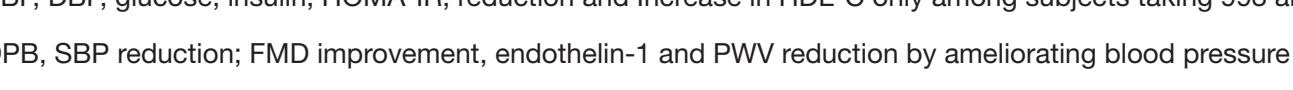

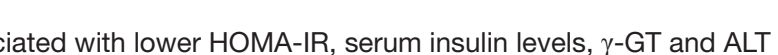

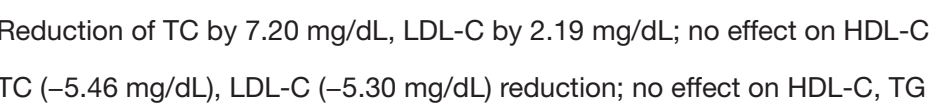

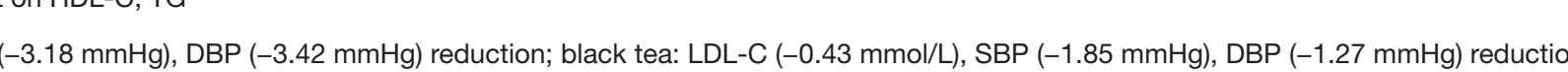

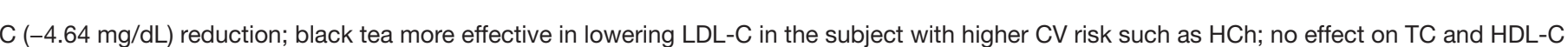

HDLCC reduction; no effect on HOL-C

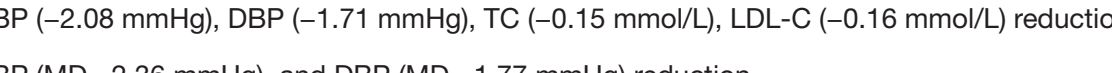

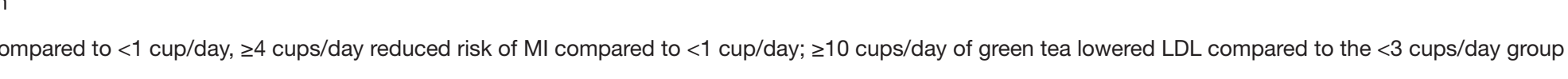

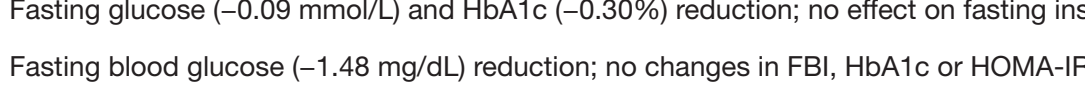

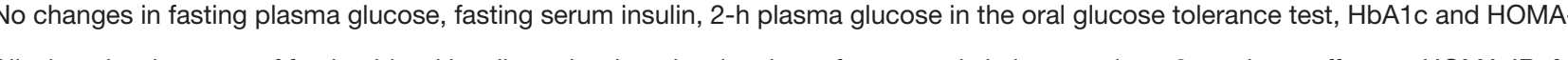

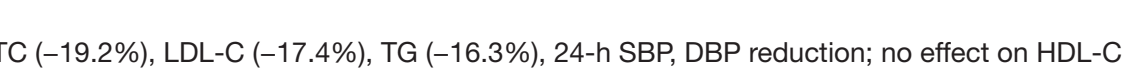

1-21\%) LDLCC (15-31\%) reduction

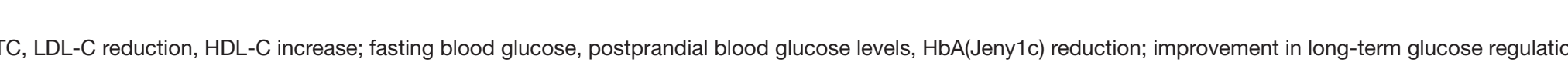

Ticeduction

soiruina, 8 g glay tor 12 weots

serulina maxima, 45 g fray for 6 wets

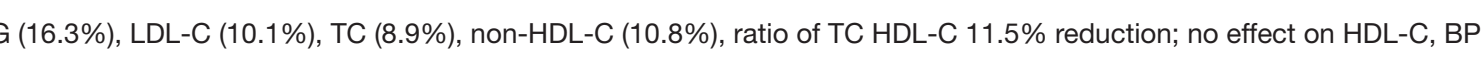

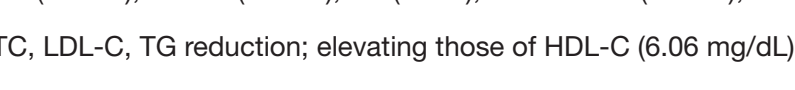

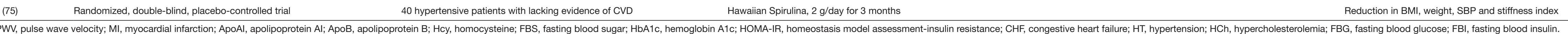

